\title{
A near Optimal Cane Rail Scheduler under Limited and Unlimited Capacity Constraints
}

\author{
Mahmoud Masoud, Erhan Kozan and Geoff Kent \\ Queensland University of Technology \\ GPO Box 2434, Brisbane Qld 4000, Australia \\ mahmoud.masoud@.qut.edu.au
}

\begin{abstract}
Australia is the world's third largest exporter of raw sugar after Brazil and Thailand, with around $\$ 2.0$ billion in export earnings. Transport systems play a vital role in the raw sugar production process by transporting the sugarcane crop between farms and mills. In 2013, 87 per cent of sugarcane was transported to mills by cane railway. The total cost of sugarcane transport operations is very high. Over $35 \%$ of the total cost of sugarcane production in Australia is incurred in cane transport.
\end{abstract}

A cane railway network mainly involves single track sections and multiple track sections used as passing loops or sidings. The cane railway system performs two main tasks: delivering empty bins from the mill to the sidings for filling by harvesters; and collecting the full bins of cane from the sidings and transporting them to the mill. A typical locomotive run involves an empty train (locomotive and empty bins) departing from the mill, traversing some track sections and delivering bins at specified sidings. The locomotive then, returns to the mill, traversing the same track sections in reverse order, collecting full bins along the way. In practice, a single track section can be occupied by only one train at a time, while more than one train can use a passing loop (parallel sections) at a time.

The sugarcane transport system is a complex system that includes a large number of variables and elements. These elements work together to achieve the main system objectives of satisfying both mill and harvester requirements and improving the efficiency of the system in terms of low overall costs. These costs include delay, congestion, operating and maintenance costs.

An effective cane rail scheduler will assist the traffic officers at the mill to keep a continuous supply of empty bins to harvesters and full bins to the mill with a minimum cost. This paper addresses the cane rail scheduling problem under rail siding capacity constraints where limited and unlimited siding capacities were investigated with different numbers of trains and different train speeds. The total operating time as a function of the number of trains, train shifts and a limited number of cane bins have been calculated for the different siding capacity constraints.

A mathematical programming approach has been used to develop a new scheduler for the cane rail transport system under limited and unlimited constraints. The new scheduler aims to reduce the total costs associated with the cane rail transport system that are a function of the number of bins and total operating costs. The proposed metaheuristic techniques have been used to find near optimal solutions of the cane rail scheduling problem and provide different possible solutions to avoid being stuck in local optima. A numerical investigation and sensitivity analysis study is presented to demonstrate that high quality solutions for large scale cane rail scheduling problems are obtainable in a reasonable time.

Keywords: Cane railway, mathematical programming, capacity, metaheuristics 


\section{INTRODUCTION}

Australia is the world's third largest exporter of raw sugar after Brazil and Thailand, with around \$2.0 billion in export earnings. Approximately $85 \%$ of the raw sugar produced in Queensland is exported. There are approximately 4400 cane farming entities growing sugar cane on a total of 380,000 hectares annually, supplying 24 mills, owned by 8 separate milling companies. Up to 35 million tonnes of sugarcane produce up to 4.5 million tonnes of raw sugar, 1 million tonnes of molasses and 10 million tonnes of bagasse annually. The sugar industry directly employs about 16,000 people across the growing, harvesting, milling and transport sectors (Australian Sugar Milling Council 2014). Transport systems play a vital role in the raw sugar production process by transporting the sugarcane crop between farms and mills. The sugarcane transport system uses a daily schedule of runs to meet the needs of both the harvesters and the mill.

Three transport methods are used to transport the cane between mills and farms; direct rail, direct road and combined road/ rail. In 2013, 87 per cent of sugarcane was transported to mills by direct rail. Direct road transport is the next most significant transport method, used to transport $8 \%$ of sugar cane. A further 5 per cent was transported by a combination of road and rail (Australian Sugar Milling Council 2014). The total cost of sugarcane transport operations is very high. Over $35 \%$ of the total cost of sugarcane production in Australia is incurred in cane transport.

A cane railway network mainly involves single track sections and multiple track sections used as passing loops or sidings. The cane railway system performs two main tasks: delivering empty bins from the mill to the sidings for filling by harvesters; and collecting the full bins of cane from the sidings and transporting them to the mill. A typical locomotive run involves an empty train (locomotive and empty bins) departing from the mill, traversing some track sections and delivering bins at specified sidings. The locomotive then returns to the mill, traversing the same track sections in reverse order, collecting full bins along the way. In practice, a single track section can be occupied by only one train at a time, while more than one train can use a passing loop (parallel sections) at a time.

The sugarcane transport system is a complex system that includes a large number of variables and elements. These elements work together to achieve the main system objectives of satisfying both mill and harvester requirements and improving the efficiency of the system in terms of low overall costs. These costs include delay, congestion, operating and maintenance costs. Integration of the harvesting, transport and milling elements in the value chain of the Australian sugar industry can increase the performance efficiency of the system. For example, optimising the delivery and collection times throughout the rail system requires information about harvester start times, harvesting rate, the number of harvesters in the system, their locations and the mill crushing rate.

Producing efficient schedules for the cane rail transport system can reduce the cost and limit the negative effects that this system can have on the raw sugar production system. A railway system is very complicated (Hu et al., 2013), especially where there is only a single track. Many publications have discussed and solved problems in this sector to improve the efficiency of the system's performance. The scheduling of locomotive movements on cane railways has proven to be a very complex task (Masoud et al., 2015). Various optimisation methods (Li et al., 2014), have been used over the years to try to produce an optimised schedule that eliminates or minimises bin supply delays to harvesters and the factory, while minimising the number of locomotives, locomotive shifts and cane bins, and also the cane age.

In this paper, a cane rail scheduler is presented that will assist the traffic officers at the mill to keep a continuous supply of empty bins to harvesters and full bins to the mill with a minimum cost. This paper addresses the cane rail scheduling problem under rail siding capacity constraints where limited and unlimited siding capacities were investigated with different numbers of trains and different train speeds. The total operating time as a function of the number of trains, train shifts and a limited number of cane bins have been calculated for the different siding capacity constraints. An adapted simulated technique was used as a metaheuristic technique with a train neighbourhood technique to solve the proposed model. 


\section{THE SUGARCANE RAIL MODEL}

A mathematical programming approach has been used to develop a new scheduler for the cane rail transport system under limited and unlimited constraints. The developed model is for a single track railway which is comprised of track sections and segments. A track section is a length of track between two adjacent points in the rail network. A track segment is a length of each branch or line and includes a set of connected sections. In the developed model, each train activity contains a series of operations defined by the start time and the processing time in a particular track section (Masoud et al. (2011)). Some track sections contain parallel tracks, typically passing loops or sidings that may or may not be suitable as passing loops.

\section{Parameters:}

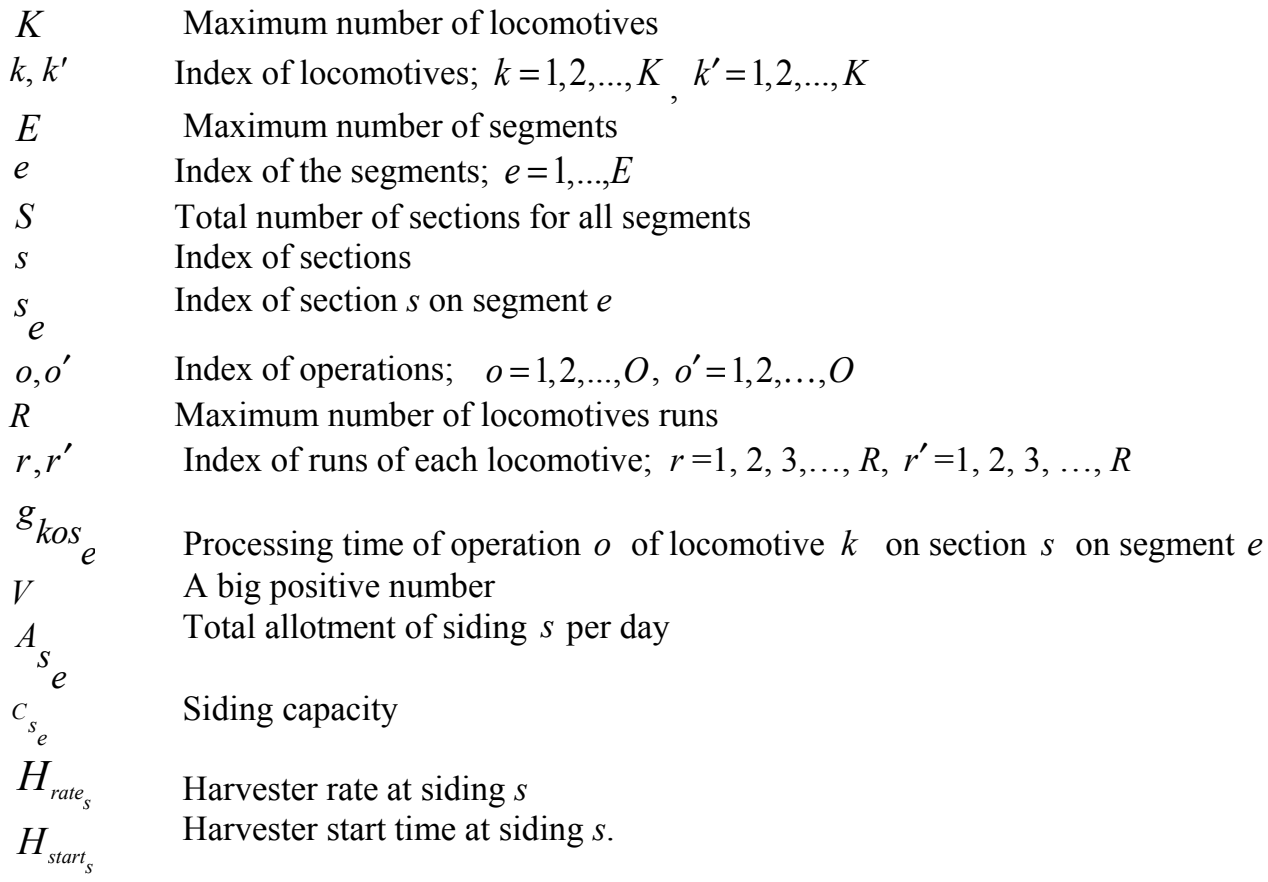

\section{Variables:}

$t_{k}$ os ${ }_{e}$

Start time of locomotive $k$ in run $r$ for operation $o$ on section $S$ on segment $e$

$B_{r} o s_{e}$

Number of full bins collected from siding $s$ by locomotive $k$ during operation $o$ and run $r$ on segmente

$\alpha_{k_{\text {os }} \text { os }} \quad$ Number of empty bins delivered for siding $s$ by locomotive $k$ during operation $o$ and run $r$ on segment $e$

${ }^{X}{ }_{r}{ }_{e} \quad=\left\{\begin{array}{l}1, \text { if locomotive } k \text { assigned to section } s \text { on segment } e \text { during run } r \\ 0, \text { otherwise. }\end{array}\right.$

$Z_{r^{\prime}} k_{r}^{\prime} s_{e}=\left\{\begin{array}{l}1, \text { if } k \text { and } n \text { are processed on section } s \text { on segment } e \text { during run } r \text { and } r^{\prime} \text { respectively, } \\ \text { and locomotive } k \text { precedes locomotive } k^{\prime} \\ 0, \text { otherwise. }\end{array}\right.$

$\beta_{k}{ }_{r} e^{s^{\prime} e^{\prime}}=\left\{\begin{array}{l}1, \text { if locomotive } k \text { uses segment } e \text { before segment } e^{\prime} \text { during run } r \\ 0, \text { otherwise. }\end{array}\right.$ 
$\lambda_{r} \quad=\left\{\begin{array}{l}1, \text { if run } r \text { is assigned for locomotive } k \\ 0, \text { otherwise. }\end{array}\right.$

$q_{r} o s_{e}=\left\{\begin{array}{l}1, \text { if the operation } o \text { of locomotive } k \text { requires section } s \text { during run } r \text { on segment } e \\ 0, \text { otherwise. }\end{array}\right.$

$U_{k_{R}^{o s} e^{s}}=\left\{\begin{array}{l}1, \text { there is a delivery at the siding } \\ 0, \text { otherwise. }\end{array}\right.$

$N_{k k^{\prime} e o s}=\left\{\begin{array}{l}1, \text { if loco } k \text { delivers } \\ 0, \text { otherwise. }\end{array}\right.$

$L_{k k^{\prime} e o s}=\left\{\begin{array}{l}1, \text { if loco collects } \\ 0, \text { otherwise. }\end{array}\right.$

$C_{\max } \quad$ Makespan.

Minimise: $C_{\max }$

Equation (2) ensures operation $o+1$ of locomotive $k$ cannot be processed before finishing operation $o$ for locomotive $k$ on section $s$ on segment $e$.

$\left(t_{k_{r} o s}+g_{k o s}\right) q_{k_{r} o s} \lambda_{k_{r}} \leq t_{k_{r}}(o+1) s_{e} q_{k_{r}}(o+1) s_{e} \lambda_{k_{r}} \forall k, r, o, s, e$

Equations (3) and (4) ensure locomotives $k$ and $k^{\prime}$ are processed on section $s$ of segment $e$ in the correct order. Either locomotive $k$ precedes locomotive $k^{\prime}$ where locomotive $k^{\prime}$ can't use this segment before locomotive $k$ leaves it, or locomotive $k^{\prime}$ precedes locomotive $k$ where locomotive $k$ can't use this segment before locomotive $k^{\prime}$ leaves it.

$$
\begin{aligned}
& t_{k_{r^{\prime}}^{\prime} o^{\prime} s_{e}} \geq t_{k_{r} o s_{e}}+\sum_{o=1}^{O} \sum_{s=1}^{S} g_{k o s_{e}}+V\left(Z_{k_{r}} k_{r^{\prime}}^{\prime} e_{e}-1\right) \\
& \forall k, k^{\prime} \in K, k \neq k^{\prime}, o, o^{\prime} \in O, s \in S_{e}, e \in E, r, r^{\prime} \in R . \\
& t_{k_{r} o s_{e}} \geq t_{k_{r^{\prime}}^{\prime} o^{\prime} s_{e}}+\sum_{o^{\prime}=1}^{O} \sum_{s=1}^{S} g_{k^{\prime} o^{\prime} s_{e}}-V\left(Z_{k_{r}} k_{r^{\prime} s_{e}}\right) \\
& \forall k, k^{\prime} \in K, k \neq k^{\prime}, o, o^{\prime} \in O s \in S_{e}, r, r^{\prime} \in R .
\end{aligned}
$$

Equation (5) ensures the finish time of each operation for all locomotives during all runs is less than the makespan.

$$
\sum_{r=1}^{R} \sum_{e=1}^{E} \sum_{s=1}^{S}\left(t_{k_{r}} O_{s}+g_{k} O_{s}\right) q_{k_{r}} O s \lambda_{e} \lambda_{r} \leq C_{\max }
$$

Equation (6) defines the blocking constraint at each segment, where, if the segment is used by locomotive $k$, all locomotives have to wait until that segment is free before they can use it.

$$
\begin{aligned}
& \sum_{k^{\prime}=1}^{K} \sum_{e=1}^{E} \sum_{s=1}^{S} t_{k_{r^{\prime}}^{\prime} o^{\prime} s_{e}} b_{k_{r}} k^{\prime} s_{e} q_{k_{r^{\prime}}^{\prime} o^{\prime} s_{e}} \lambda_{k_{r^{\prime}}} \geq \sum_{e=1}^{E} \sum_{s=1}^{S} q_{k_{r} o s}{ }_{e} k_{r} o s \\
& \forall k, r, r^{\prime}, o, o^{\prime} .
\end{aligned}
$$

Equations (7) and (8) show the relation between the total bin allotments for each siding and the number of empty and full bins delivered to and collected from each siding. The total number of empty bins delivered to siding $s$ has to be equal to the allotment of the harvester operating at this siding.

$\sum_{k=1}^{K} \sum_{r=1}^{R} \sum_{e=1}^{E} \sum_{o=1}^{O} q_{k_{r} o s_{e}} \alpha_{k_{r} o s_{e}} \lambda_{k_{r}}=A_{s_{e}} \forall s$. 
The total number of full bins collected from siding $s$ has to be equal to the allotment of the harvester operating at this siding.

$$
\sum_{k=1}^{K} \sum_{r=1}^{R} \sum_{e=1}^{E} \sum_{o=1}^{O} q_{k_{r} o s_{e}} B_{k_{r} o s_{e}} \lambda_{k_{r}}=A_{s_{e}} \forall s .
$$

Equation (9) ensures that the total number of empty and full bins at each siding is less than the capacity of the siding.

$$
\alpha_{k_{r} o s_{e}}+B_{k_{r} o s_{e}} \leq \mathrm{C}_{s_{e}}
$$

Equation (10) ensures the number of delivered empties in the last run is greater than or equal to the time of the first run and the harvester start, considering the harvesting rate for each harvester.

$$
B_{k_{R^{o} e^{s} e}} \geq\left(\left(t_{k_{1} e_{e}^{s} e}-\mathrm{H}_{\mathrm{start}_{\mathrm{s}}}\right) /(60 * 60)\right) * \mathrm{H}_{\mathrm{rate}_{\mathrm{s}}}-M * U_{k_{R^{o}}^{o} e^{s}}
$$

Equations (11) and (12) ensure there is no delay time in delivering to the harvesters during the visits, where the time of the second visit for each siding depends on the number of empty bins delivered in the first visit, and so on. Equations (11) and (12) ensure the delivered empty bins in any run will be sufficient until the next visit. This equation works from the second visit to the last visit for each siding.

$$
\begin{aligned}
& B_{k_{r} e^{s} e} \leq M^{*}\left(1-U_{k_{r} e^{s} e^{s}}\right) \\
& B_{k_{r} e^{s} e^{s}} \leq\left(\left(t_{k_{(r+1)} e^{s} e^{s}}-t_{k_{r} o_{e}^{s} e}\right) /(60 * 60)\right) * \mathrm{H}_{\text {rates }}-M * U_{k_{r} e^{s} e_{e}}
\end{aligned}
$$

The number of full bins collected depends on the harvesting rate at each siding and the visit times. The Equations (13) and (14) ensure that the number of full bins collected is no more than the produced number of full bins at each siding.

$$
\begin{aligned}
& \left.\alpha_{k_{1} o_{e}^{s} e} \leq\left(t_{k_{1} e_{e}^{s} e}-\mathrm{H}_{\mathrm{start}_{\mathrm{s}}}\right) /(60 * 60)\right) * \mathrm{Hrate}_{\mathrm{s}} \\
& \alpha_{k_{r^{o} e^{s} e}} \leq\left(\left(t_{k_{(r+1)} e_{e} e_{e}}-t_{k_{r^{o} e^{s} e}}\right) /(60 * 60)\right) * \mathrm{Hrate}_{\mathrm{s}}
\end{aligned}
$$

\section{SOLUTION APPROACH}

An adapted simulated annealing (SA) algorithm was developed to solve the sugarcane rail transport problem. Firstly, initial feasible solutions are obtained and then the quality of the solutions is improved. This algorithm depends on using a neighbourhood structure that depends on swapping any two adjacent trains at any junction point through rail network. Many different neighbourhoods will be produced to improve the final solution.

Select the initial simulated annealing parameters

Define a large value for the temperature $T=T_{0} ; T_{0}=100$.

Select value of the parameter $\alpha ; 0<\alpha<1 ; \alpha=0.95$

Construct an initial solution using First Come First Service, FCFS.

Define initial scheduling (s) for all trains on all sections on first segment

Define initial sequencing of segments( construct an initial order of segments to be used by each train)

Calculate initial makespan (currMakespan)

While (T is in cooling range)

Construct a new neighbourhood

Apply Adjacent two trains technique

Select $i, j$ Es, where $i, j$ are two adjacent trains on a junction point. (Step 1)

Swap $i$ and $j$ trains that require a specific segment and a specific section Store the new schedule s'

Check the feasibility

Time window, section transition matrix, trains and sidings capacity

Keep the sequencing of sections without changing

Produce a new schedule and a new solution (new Makespan)

Evaluate the new makespan where $\Delta=n e w$ Makespan-currMakespan

If $\Delta<0$ then 
Accept s' as a new schedule

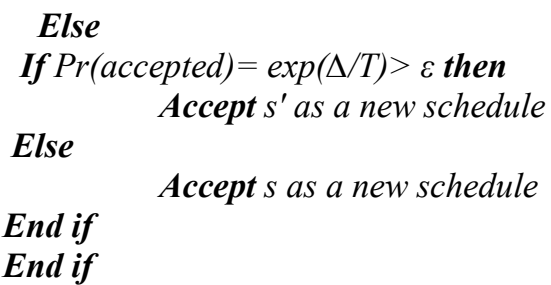

Update T according to $T=\alpha T$

End while

Stop criteria $\Delta$ is not significant or there are no improvements in the makespan for small change in $T$ End.

In this paper, the initial temperature, $\mathrm{T}_{0}=100$, and the $\mathrm{SA}$ parameter, $\alpha=0.95$, were used as the SA parameters benchmark to produce the SA solution. The SA parameter, $\alpha=0.95$, was selected by comparing the makespan value under this value and other common values such as 90, 92 and 98 values, Masoud et. al.(2015). The initial solution was constructed to be used in the SA procedure using First Come First Service, FCFS, where each train was served at each section depending on the start time at this section. The difference between current makespan and previous makespan will be used to measure the value of the improvement in the SA procedure. The adjacent two trains technique is used to produce different neighbourhoods at each conjunction or intersection point of more than one segment. This technique depends on the order of number of operations of some trains that require same section at a time. For example, two trains, 1 and 2 arrived at intersection point at rail and all require same segment. Neighbourhoods are the different order of trains on same segment such as $1-2$ or $2-1$.

\section{IMPLEMENTATION}

In practice, many factors affect the efficiency of the cane rail system such as the number of trains that can be used in the system to satisfy the harvester and mill constraints, and siding capacity constraints. In this paper, two main objectives, makespan (finish time of the last operation of locomotives on the railway system) and total operating time of the locomotives are used to conduct a sensitivity analysis of these factors on the efficiency of the cane rail system. In the current case study, there are 87 track sections and harvesters are operating at 33 active sidings across three days utilising 4789 bins as a total allotment. Not all sidings are active each day. The maximum acceptable makespan of the system is 3 days ( 72 hours). The model was solved with 2 to 9 trains to calculate the makespan and the total operating time. Figure 1 shows the makespan in hours, number of runs and total operating times in hours for different number of trains where the trains speed is a fixed value, $30 \mathrm{~km} / \mathrm{h}$, and the capacity of the sidings is limited. The makespan of 2 trains is 95.0 hours, the total operating time equals 156 hours and 72 runs were required. While the total operating time is low, the makespan is greater than the maximum makespan of the system ( 72 hours). As a result, using the 2 trains in the system is not sufficient to satisfy the total allotment and the 2 trains case is not acceptable. Increasing to 3 or 4 trains still results in the makespan being greater than 72 hours.

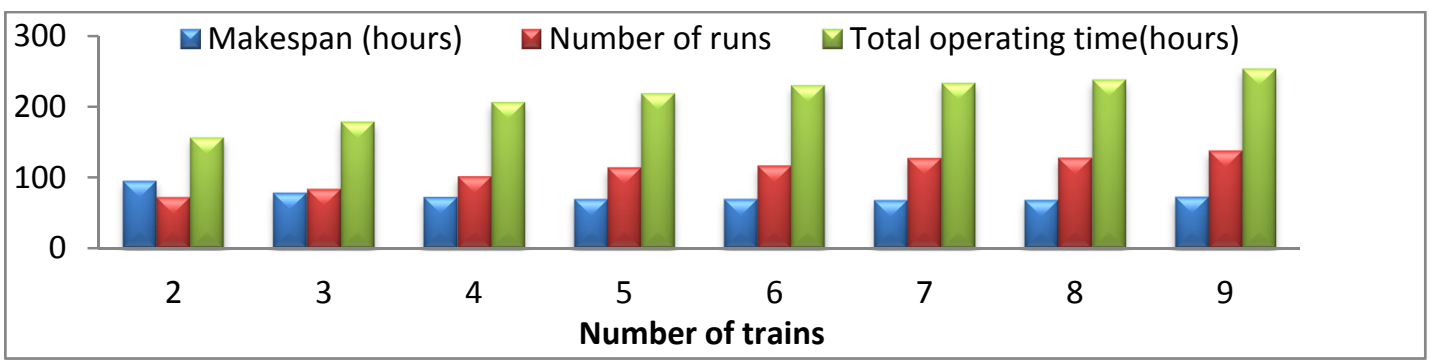

Figure 1. Makespan and total operating time using different number of trains.

The 5 train case provided one of the lowest makespans ( 69.8 hours) and total operating time (218 hours) with a total of 114 runs. This case has the lowest number of trains of the feasible solutions. The 8 train case had the lowest makespan (68.5 hours), 1.3 hours less than the 5 train case but did it with considerably higher total 
operating time and number of runs. In real life using ACRSS, the current model satisfy around more than $15 \%$ improvement by using ACRSS software (automatic cane railway scheduling system) in the total operating time of 6 locomotives. The effects of the train speed changes on the total operating time and the makespan were studied for the 5 train case for speeds from $27 \mathrm{~km} / \mathrm{h}$ to $45 \mathrm{~km} / \mathrm{h}$. Figure 2 shows the results. The best results were obtained for a train speed of $42 \mathrm{~km} / \mathrm{h}$, with a makespan of 68.3 hours and the smallest total operating time of 196 hours. The solutions for 27,28 and $32 \mathrm{~km} / \mathrm{h}$ were not feasible since their makespan was greater than 72 hours

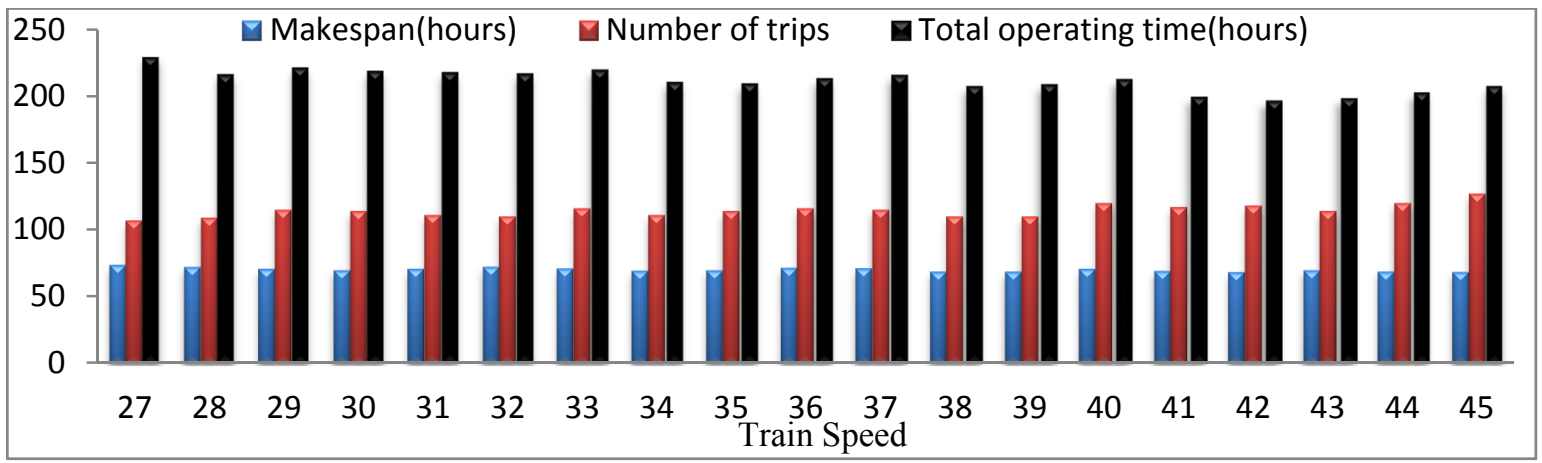

Figure 2. The makespan, number of trips and the total operating time under different train speeds.

Each siding has a limited space to store specific number of bins (the siding capacity) or unlimited capacity. Siding capacity has a big effect on the cane train schedules. To determine the effect of the siding capacity constraints on the efficiency of the cane rail system, schedules were produced for limited and unlimited siding capacity. Compared to the limited siding capacity case that had a makespan of 69.8 hours, a total operating time of 218 hours and 114 runs, the unlimited siding case produced a makespan of 68.6 hours, total operating time of 208 hours and 115 runs. The unlimited siding capacity reduced the makespan by $1.7 \%$ and the total operating time by $4.9 \%$.

\section{CONCLUSION}

The proposed scheduling model is very complicated and needs to be solved in a reasonable time, because of the dynamic nature of the system and large number of variables. In the developed model, the system constraints can be classified into two main categories. Firstly, there are constraints related to the rail operations system. These constraints are very important to ensure passing of trains without accidents or delays. Secondly, there are constraints related to cane rail operations such as the train, siding and mill capacity, empty bins requirements, and full bin requirements. Adapted simulated annealing was used to solve the proposed model to evaluate different objectives such as minimising total operating time, makespan and number of runs in the cane rail system. In this paper, the model examined the effect of changing the number of locomotives, train speed and siding capacity. For the model studied, 5 trains provided the best overall result with low makespan and total operating time. Higher speed generally gave better results than lower speed. Unlimited siding capacity improved the makespan and total operating time by $1.7 \%$ and $4.9 \%$ respectively.

\section{REFERENCES}

Hu, H, Li, K. \& Xu, X. (2013). A multi-objective train-scheduling optimization model considering locomotive assignment and segment emission constraints for energy saving, Journal of Modern Transportation, 21 (1), 916.

Li, X, Chien, C.F, ,Yang, L.X, \& Gao, Z. (2014). The train fuelling cost minimization problem with fuzzy fuel prices, Flexible Service and Manufacturing Journal, 26, 249-267.

Masoud, M., Kozan, E. \& Kent, G.A. (2011). A job-shop scheduling approach for optimising sugarcane rail operations. Flexible Services and Manufacturing Journal, 23 (2), 181-196.

Masoud, M., Kozan, E. \& Kent, G.A. (2015). Hybrid metaheuristic techniques for optimising sugarcane rail operations. International Journal of Production Research, 53(9), 2569-2589. 